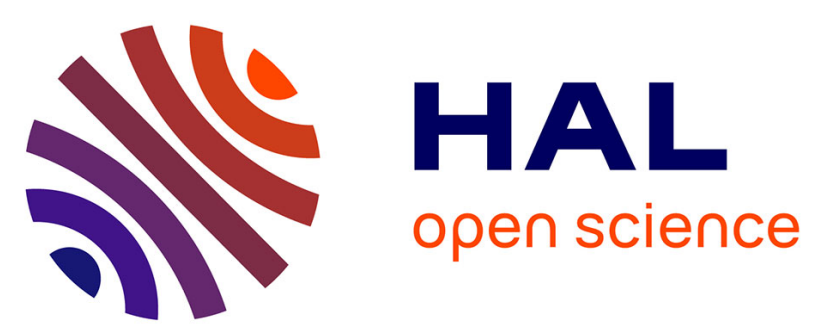

\title{
SIMULATION NUMÉRIQUE DE LA PROPAGATION D'UNE ONDE ACOUSTIQUE DANS UNE TURBULENCE BIDIMENSIONNELLE PAR UNE MÉTHODE DE RAYONS
}

P. Blanc-Benon, D. Juvé

\section{To cite this version:}

P. Blanc-Benon, D. Juvé. SIMULATION NUMÉRIQUE DE LA PROPAGATION D'UNE ONDE ACOUSTIQUE DANS UNE TURBULENCE BIDIMENSIONNELLE PAR UNE MÉTHODE DE RAYONS. Journal de Physique Colloques, 1990, 51 (C2), pp.C2-1149-C2-1152. 10.1051/jphyscol:19902269 . jpa-00230600

HAL Id: jpa-00230600 https://hal.science/jpa-00230600

Submitted on 1 Jan 1990

HAL is a multi-disciplinary open access archive for the deposit and dissemination of scientific research documents, whether they are published or not. The documents may come from teaching and research institutions in France or abroad, or from public or private research centers.
L'archive ouverte pluridisciplinaire HAL, est destinée au dépôt et à la diffusion de documents scientifiques de niveau recherche, publiés ou non, émanant des établissements d'enseignement et de recherche français ou étrangers, des laboratoires publics ou privés. 
COLLOQUE DE PHYSIQUE

Colloque C2, supplément au $n^{\circ} 2$, Tome 51, Février 1990

ler Congrès França1s d'Acoustique 1990

\title{
SIMULATION NUMERIQUE DE LA PROPAGATION D'UNE ONDE ACOUSTIQUE DANS UNE TURBULENCE BIDIMENSIONNELIE PAR UNE METHODE DE RAYONS
}

\author{
P. BLANC-BENON et D. JUVE \\ Laboratoire de Mécanique des Fluides et d'Acoustique, CNRS URA 263. \\ Ecole Centrale de Lyon, BP. 163, F-69131 Ecully Cedex, France
}

Résumé : Nous présentons des résullats de simulation numérique de la propagation d'une onde acoustique dans une turbulence cinématique bidimensionnelle. Le milieu turbulent est représenté par une superposition de modes de Fourier aléatoires. Pour chaque réalisation, on détermine les rayons issus d'une source ponctuelle, on calcule la variance du temps de parcours le long de ces trajectoires ainsi que la distance pour laquelle se forme la première caustique.

Abstract: A numerical technique for simulating the behavior of an acoustic wave propagating through a turbulent medium is presented. A bidimensional velocity field is generated using a superposition of random Fourier modes. The time variance along the rays and the probability density for the occurence of the first caustics are computed.

\section{1 - INTRODUCTION}

L'effet de la turbulence sur la propagation des ondes est un problème dont l'analyse théorique a été envisagée par de nombreux auteurs avec des applications importantes en acoustique aérienne ou sous-marine. Les fluctuations aléatoires de vitesse ou de température du milieu de propagation modifient les propriétés spatiotemporelles des fronts d'onde transmis. On observe alors notamment des variations importantes des temps de parcours de l'onde entre la source et une antenne de récepteurs.

Les différentes approches théoriques disponibles dans la littérature (Chernov /1/, Tatarski /2/, Flatte /3/, Uscinski /4/) reposent sur l'écriture d'une équation d'onde dont la résolution nécessite une modélisation des effets d'interaction entre l'onde et le milieu turbulent. Compte tenu des hypothèses nécessaires pour établir ces formulations (analytiques ou numériques), il est difficile d'en préciser le domaine de validité. Nous présentons ici une simulation numérique développée à partir d'une représentation d'un milieu turbulent par une superposition de modes de Fourier aléatoires. La répartition spectrale de ces modes ainsi que l'évolution de leur amplitude sont choisies de façon à réaliser une turbulence de caractéristiques statisliques données (valeurs efficaces des fluctuations, échelles de corrélations spatiales, spectre d'énergie). Pour chacune des réalisations, on détermine les rayons issus d'une source ponctuelle. Les résultats présentés ici concernent la propagation dans une turbulence cinématique bidimensionnelle. En particulier on donne l'évolution de la variance du temps de parcours le long des rayons ainsi que la distance moyenne de formation de la première caustique.

\section{2 - SIMULATION D'UNe TURBULENCE CINEMATIQUE BIDIMENSIONNELLE. hOMOgENE ET ISOTROPE}

En reprenant une démarche introduite par Kraichnan $15 /$, nous simulons une réalisation d'un champ de vitesse $\vec{V}(\vec{X})$ d'une turbulence homogène et isotrope en superposant $N$ modes de Fourier aléatoires $\vec{U}(\overrightarrow{\mathrm{K}})$ :

$$
\text { (1) } \quad \overrightarrow{\mathrm{V}}(\overrightarrow{\mathrm{X}})=\sum_{\mathrm{l}=1}^{N} \overrightarrow{\mathrm{U}}\left(\overrightarrow{\mathrm{R}}^{1}\right) \cos \left(\overrightarrow{\mathrm{R}}^{1} \cdot \overrightarrow{\mathrm{X}}+\psi^{l}\right)
$$

La direction du vecteur $\vec{K}^{1}$ est choisie aléatoirement et équirépartie dans le plan $\left(\vec{K}_{1}, \vec{K}_{2}\right)$ afin d'assurer

l'isotropie du champ. La phase $\psi^{\prime}$ est une variable aléatoire également répartie sur l'intervalle $[0,2 \pi]$, 
rendant le champ aléatoire homogène. La condition d'incompressibilité, $\operatorname{div}(\vec{V})=0$, est respectée en choisissant le vecteur $\overrightarrow{\mathrm{U}}\left(\overrightarrow{\mathrm{K}}^{1}\right)$ perpendiculaire à $\overrightarrow{\mathrm{K}}^{1}$. L'amplitude de chaque mode $\left\|\overrightarrow{\mathrm{U}}\left(\overrightarrow{\mathrm{K}}^{1}\right)\right\|$ est reliée au spectre d'énergie cinétique $\mathrm{E}(\mathrm{K})$ et choisie de façon à obtenir une valeur efficace des fluctuations $v^{\prime}$ (Blanc-Benon et alii /6/). Pour les résultats présentés ici, nous avons une fonction de corrélation spatiale gaussienne pour le champ de vitesse $\left(f(r)=\exp \left(-r^{2} / L^{2}\right)\right)$. Le spectre $E(K)$ est alors :

$$
\text { (2) } \quad E(K)=v^{\prime 2} \frac{K^{3} L^{4}}{8} \exp \left(-\frac{K^{2} L^{2}}{4}\right)
$$

L'échelle intégrale des fluctuations de vitesse $L_{f}$ vaut alors $\frac{L \sqrt{\pi}}{2}$. Dans nos essais nous utilisons 50 modes de Fourier répartis entre $K=1 \mathrm{~m}^{-1}$ et $K=100 \mathrm{~m}^{-1}$, avec une échelle $L$ de $10 \mathrm{~cm}$.

\section{3 - equations des rayons}

Dans la théorie géométrique, les rayons acoustiques sont obtenus en cherchant les lignes caractéristiques associées à la relation de dispersion. En considérant que le temps de iraversée de la turbulence est neltement inférieur au temps d'évolution de struclures turbulentes, nous faisons l'hypothèse de turbulence "gelée". Pour un milieu en mouvement, indépendant du temps, le problème de propagation est décrit à partir des solutions du système d'équations différentielles suivant (Candel $/ 7 /$ ):

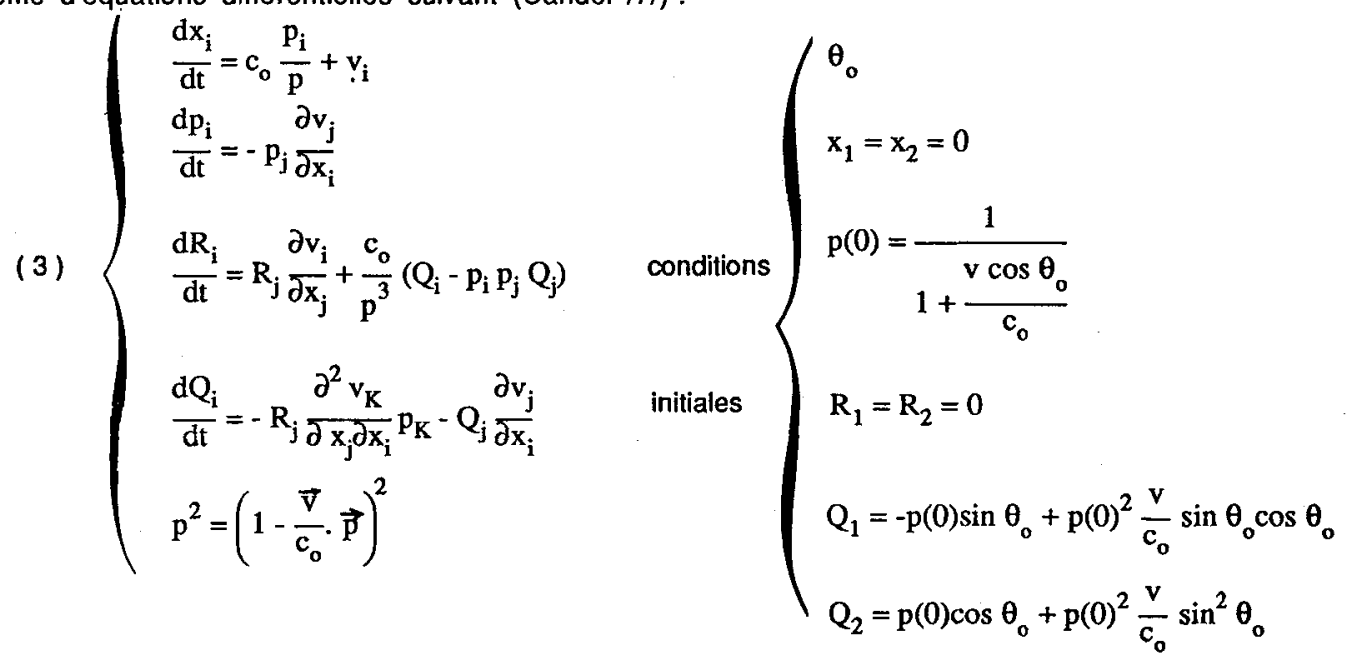

La position $\vec{X}\left(S, \theta_{0}\right)$ et le vecteur d'onde adimensionnel $\vec{p}\left(S, \theta_{0}\right)$ du point courant d'un rayon acoustique sont fonction uniquement de l'angle initial $\theta_{0}$ el de la phase $S=c_{0} t \quad\left(c_{0}\right.$ célérité du son dans le milieu au repos), et sont donnés par les quatre premières équations différentielles du système $/ 3 /$. Les vécteurs $\vec{R}=\left(\frac{\partial \vec{X}}{\partial \theta_{0}}\right)_{S}$ et $\vec{Q}=\left(\frac{\partial \vec{p}}{\partial \theta_{0}}\right)_{S}$ permettent de caractériser le front de phase le long des trajectoires, et l'évolution de la section droite d'un tube élémentaire de rayon. En chercnant les conditions d'annulation de cette section droite, il est possible de localiser les caustiques et de déterminer la distance de formation de la première d'entre elles. Le système différentiel (3) est intégré avec une méthode de Runge-Kutta d'ordre 4 avec un pas de temps $\Delta t=\left(c_{0} K_{\max }\right)^{-1},\left(K_{\max }\right.$ nombre d'onde maximum utilisé dans la modélisation de $\left.E(K)\right)$. 
Sur la figure 1 nous avons reporté deux tracés de rayons obtenus pour deux réalisations du champ de vitesse avec la même échelle de corrélation spatiale $L=10 \mathrm{~cm}$. On observe une distorsion des rayons importante et l'apparition de caustiques pour le cas $v^{\prime}=2 \mathrm{~m} / \mathrm{s}$ à $X=2 \mathrm{~m}$ environ. Sur la figure 2 nous avons tracé l'évolution de la variance du temps de parcours $\left\langle t^{\prime 2}\right\rangle$ pour des rayons issus d'une source ponctuelle allant à une distance fixe R. A titre de comparaison nous avons reporté également la solution de Chernov donnée par la relation :

$$
\text { (4) }\left\langle\mathrm{t}^{\prime 2}\right\rangle=2 \frac{\mathrm{v}^{\prime 2}}{\mathrm{c}_{\mathrm{o}}^{4}} \mathrm{R} \mathrm{L}_{\mathrm{f}}
$$

On observe qu'après une zone de croissance linéaire, en accord avec les prédictions théoriques de Chernov, la variance du temps de parcours croît de façon très marquée, notamment pour $v^{\prime}=2 \mathrm{~m} / \mathrm{s}$. Un résultat analogue a également été obtenu pour une turbulence cinématique tridimensionnelle modélisée par un spectre de von Karman (Blanc-Benon el alii $/ 6 /, / 8 /$ ). Afin d'analyser l'origine de cet écart, nous avons calculé la distance de formation de la première caustique. La densité de probabilité normalisée d'apparition de la première caustique est donnée sur la figure 3 pour le cas $v=2 \mathrm{~m} / \mathrm{s}$. Le maximum de celle densité de probabilité est obtenu pour un rapport $R / L$ d'environ 20 ce qui correspond à la distance pour laquelle la variance $\left\langle\mathrm{t}^{\prime 2}\right\rangle$ ne suit plus la loi linéaire de Chernov.

\section{5 - CONCLUSION}

La méthode de simulation par modes de Fourier aléatoires d'une turbulence cinématique homogène et isotrope introduite ici, permet de caractériser la propagation d'une onde acoustique dans un milieu turbulent sans modélisation a priori des effets d'interaction entre l'onde et le milieu. Dans le cadre des hypothèses de l'acoustique géométrique nous avons étudié la variance du temps de parcours el analysé son évolution en fonction de l'existence dans le milieu de propagation de caustiques aléatoires, précisant ainsi le domaine de validité de la solution de Chernov.

Cette méthode de simulation peut être étendue au cas de milieux turbulents plus complexes (anisotropie, inhomogénéité) ce qui devrait ainsi permettre de traiter des cas de propagation plus proches de la réalité pour lesquels peu de résultats analytiques existent.

\section{REFERENCES}

$11 /$ Chernov, L., Wave Propagation in a Random Medium, Mc Graw-Hill, New York (1960).

/ 2 / Tatarski, V.I., The Effects of the Turbulent Atmosphere on Wave propagation, I.P.S.T. Keter Press, Jerusalem (1971).

/ $3 /$ Flatte, S.M., Proc. IEEE, Z1 (11), (1983), 1267-1293.

/ 4 / Uscinski, B.J., J. Opt. Soc. Am. 2 (12), (1985), 2077-2091.

/ 5 / Kraichnan, R.H., Phys. Fluids, 13 (1), (1970), 22-31.

$16 /$ Blanc-Benon, Ph., Juvé, D., Karweit, M., Comte-Bellot, G., Journal d'Acoustique, Vol. $2 n^{\circ}$ 1, (1990), (à paraître).

$17 /$ Candel, S., J. Fluid Mech., 83, 3, (1977), 465-493.

/ 8 / Blanc-Benon, Ph., Karweit M., 13 ICA Belgrade, Vol. 1, (1989), 321-324. 

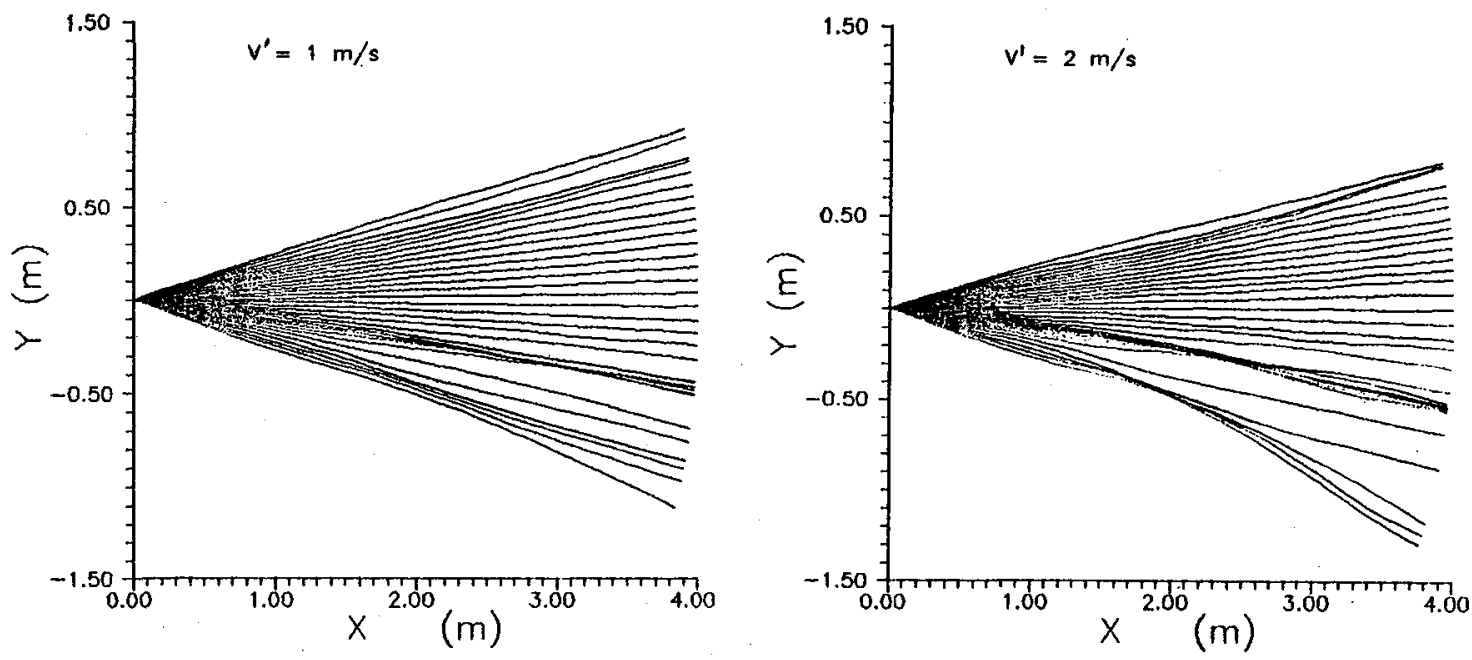

Fig. 1 : Exemples de tracé de rayons acoustiques

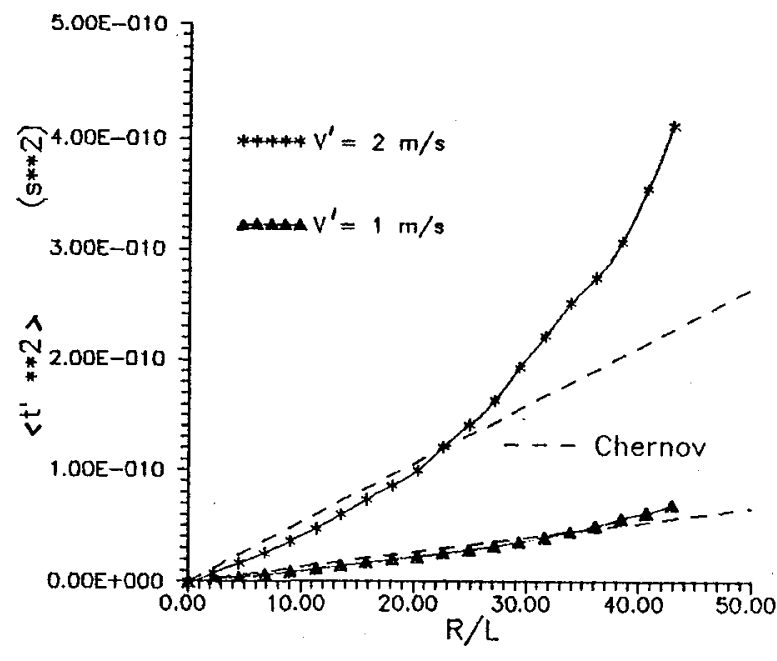

Fig. 2 : Variances du temps de parcours le long de rayons arrivant a une distance $R$ (250 réalisations; 50 modes)

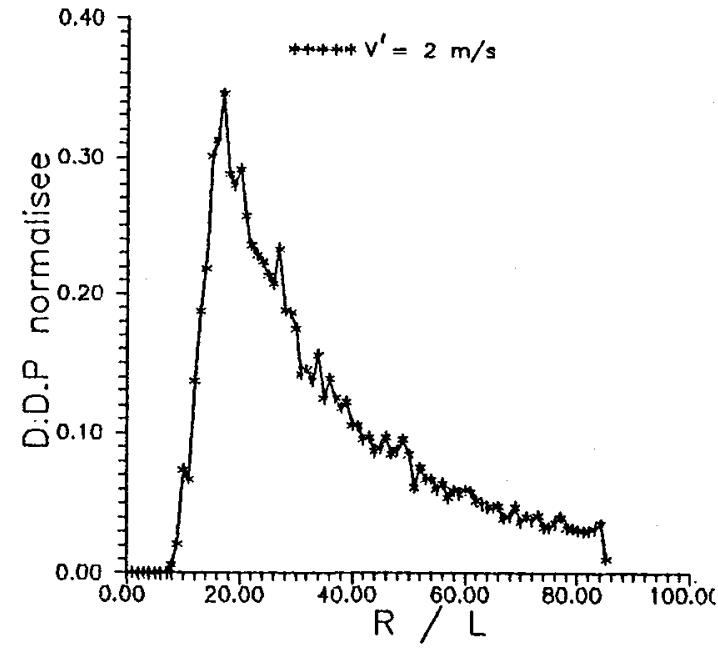

Fig. 3 : Densité de probabilité de formation de la première caustique (250 réalisations ; 50 modes) 